\section{Deficiências visual, auditiva e motora entre a população indígena no Brasil}

\author{
Visual, hearing, and motor impairments in the \\ indigenous population in Brazil
}

Discapacidad visual, auditiva y motora entre la población indígena en Brasil
Claudio Santiago Dias Junior 1

Ana Paula Verona 2

\section{Resumo}

O objetivo deste estudo foi descrever a prevalência de deficiências visual, auditiva e motora e estimar a chance de se ter uma das três deficiências, separadamente, segundo grau de severidade, para a população indígena no Brasil. Os dados foram retirados do Censo Demográfico de 2010 coletados pelo Instituto Brasileiro de Geografia e Estatística (IBGE). Os métodos utilizados incluem a padronização direta para o cálculo das prevalências e modelos de regressão logística multinomial. Os resultados padronizados mostram que homens e mulheres indígenas apresentam a maior prevalência em cada uma das deficiências examinadas neste trabalho, sendo a única exceção a deficiência visual de grau leve entre as mulheres. Os resultados dos modelos de regressão multinomial mostram uma desvantagem relativa dos povos indígenas em quase todos os tipos de deficiência.

Pessoas com Deficiência; Índios Aul-Americanos; Saúde dos Povos Indígenas

\author{
Correspondência \\ C. S. Dias Junior \\ Departamento de Sociologia, Universidade Federal de Minas \\ Gerais. \\ Av. Antonio Carlos 6627, Belo Horizonte, MG 31270-901, \\ Brasil. \\ csdj@ufmg.br \\ ${ }^{1}$ Departamento de Sociologia, Universidade Federal de Minas \\ Gerais, Belo Horizonte, Brasil. \\ 2 Centro de Desenvolvimento e Planejamento Regional, \\ Universidade Federal de Minas Gerais, Belo Horizonte, Brasil.
}




\section{Introdução}

As condições de saúde da população indígena no Brasil têm sido objeto de diversas análises, sendo que a maioria delas é focada em doenças infectoparasitárias 1,2, doenças crônicas 3,4, questões nutricionais 5,6, saúde bucal 7,8, uso de álcool e drogas 9,10, suicídio 11 e, recentemente, na mortalidade ${ }^{12}$. De uma maneira geral, a situação das condições de saúde dos povos indígenas em relação à população não indígena é extremamente desfavorável no Brasil 13,14.

É importante destacar que esses estudos têm tido um papel fundamental para delinear o perfil epidemiológico dos povos indígenas no Brasil e evidenciar as demandas de uma parcela da população que historicamente tem tido dificuldades de acesso aos bens e serviços disponibilizados pelo Estado brasileiro.

Apesar dos esforços, algumas lacunas podem ser observadas na literatura corrente sobre a saúde dos povos indígenas. Diferentemente de alguns países, como a Austrália 15, Canadá 16 e Estados Unidos 17 , é evidente a falta de estudos relacionados à prevalência das deficiências físicas entre os indígenas no Brasil. Não se sabe quais tipos de deficiência física os atinge, em qual intensidade, como se distribuem geograficamente, se e como é o acesso ao sistema de saúde para lidar com essas questões. Além disso, pouco se sabe sobre os diferenciais na prevalência ou na chance de ter deficiência física segundo cor/raça no Brasil. Tal desconhecimento é ainda maior ao se considerar a população indígena, que usualmente é retirada das análises que empregam dados populacionais.

Tentando reduzir, em parte, essa lacuna, este trabalho tem como objetivo inicial descrever as prevalências (padronizada pela estrutura etária) de deficiências visual, auditiva e motora, segundo grau de severidade, entre a população indígena e compará-las com aquelas observadas nos demais grupos de cor/raça do Brasil. Em seguida, este trabalho compara a chance de ter uma das três deficiências, separadamente e segundo grau de severidade, para cada grupo de cor/raça, controlando por outras variáveis associadas à chance de apresentar deficiência. Para estimar as razões de chance, foram utilizados modelos de regressão logística multinomial para cada tipo de deficiência, segundo grau de severidade. Os dados utilizados neste estudo foram retirados do Censo Demográfico de 2010 do Instituto Brasileiro de Geografia e Estatística (IBGE) 18. As análises foram realizadas utilizando o pacote estatístico IBM SPSS 25 (IBM Corp., Armonk, Estados Unidos).

\section{Material e métodos}

\section{Dados}

Para a realização deste estudo, foram utilizados os dados do Censo Demográfico de 2010 18. Embora o banco de dados do universo contenha a variável cor/raça, foi necessário utilizar as informações do banco de dados da amostra porque apenas esse possui tanto o quesito cor/raça quanto os quesitos sobre deficiência. Foram analisadas informações de indivíduos com cinco anos ou mais de idade, de ambos os sexos, o que totalizou uma população estimada de quase 177 milhões de pessoas. Crianças com menos de cinco anos não foram incluídas na análise devido à dificuldade de diagnóstico de deficiência inerente à idade.

\section{Variáveis}

\section{- Deficiência}

A deficiência foi captada pelo Censo Demográfico de 201018 a partir de quatro questões: capacidade do indivíduo de enxergar, ouvir, caminhar ou subir escadas (que, neste estudo, será denominada deficiência motora) e deficiência mental/intelectual. Para a realização deste estudo, foram selecionadas as três primeiras: as deficiências visual, auditiva e motora. Além da presença de deficiência, o Censo perguntou o grau de severidade. Dessa forma, foram apresentadas as seguintes opções se o indivíduo reportasse ter alguma deficiência: (1) não consegue de modo algum, (2) consegue com grande dificuldade e, (3) consegue com alguma dificuldade. Caso o indivíduo declarasse não ter deficiência, era marcada a opção: consegue sem nenhuma dificuldade. 
Para o IBGE, é classificado como deficiente o indivíduo que relatasse alguma dificuldade em enxergar, ouvir, andar ou subir escadas, mesmo que usando algum aparelho corretivo, como óculos, aparelhos auditivos, próteses, dentre outros.

Como a variável de análise é a presença (ou ausência) de deficiência (visual, auditiva ou motora) segundo grau de severidade, este trabalho criou uma interação entre essas informações, utilizando, assim, as seguintes categorias para a variável de análise: (1) deficiência grave: agrupa os indivíduos que reportaram que não conseguem ou têm grande dificuldade de enxergar, ouvir ou caminhar ou subir escadas; (2) deficiência leve: inclui os indivíduos com alguma dificuldade de enxergar, ouvir ou caminhar ou subir escadas e; (3) não possui deficiência.

\section{- Cor/raça}

O Censo Demográfico de 201018 coletou a informação sobre cor/raça a partir do quesito "a sua cor ou raça é?". É importante mencionar que o Censo Demográfico de 2010 avançou na captação da informação sobre os indígenas com o quesito "você se considera indígena?", que era respondido por moradores de Terras Indígenas que não se classificaram como indígena no quesito "a sua cor ou raça é?". Embora exista a possibilidade de agrupar na categoria indígena os respondentes desses dois quesitos, este trabalho utilizou como base de informação apenas o quesito "a sua cor ou raça é".

A variável cor/raça do Censo Demográfico de 2010 foi categorizada da seguinte maneira: (1) branca, (2) preta, (3) amarela, (4) parda e (5) indígena.

\section{Variáveis-controle}

Foram utilizadas como variáveis-controle: idade, sexo, escolaridade do chefe do domicílio, região de residência e situação de domicílio. A variável sexo foi categorizada em (1) masculino e (2) feminino. A partir da variável idade completa, foram criados grupos etários de 5-14 anos, 15-59 anos e 60 anos e mais. A variável de escolaridade do chefe do domicílio, usada neste estudo, foi categorizada pelo Censo da seguinte maneira: (1) sem instrução/Ensino Fundamental incompleto; (2) Ensino Fundamental completo/Ensino Médio incompleto; (3) Ensino Médio completo/Ensino Superior incompleto e (4) Ensino Superior completo. Além dessas, foram utilizadas as variáveis de situação do domicílio, categorizada em (1) rural e (2) urbano e a de região geográfica, que é categorizada em (1) Norte, (2) Nordeste, (3) Sudeste, (4) Sul e (5) Centro-oeste.

A escolha das variáveis-controle foi feita segundo revisão da literatura prévia que indicava as principais variáveis associadas à deficiência física no Brasil e no contexto internacional. A literatura corrobora a relação entre sexo, idade, educação do chefe do domicílio além de aspectos relacionados ao local de residência e à ocorrência de algum tipo de deficiência física 16,19,20. De uma maneira geral, mulheres, pessoas mais velhas e residentes em domicílios cujo responsável tem baixa escolaridade apresentam maiores chances de possuir deficiências. Já o local de residência pode fazer com que os indivíduos tenham um maior acesso ou não aos serviços de saúde e, por consequência, um diagnóstico melhor sobre as questões relacionadas com a saúde 15,18,21.

\section{Métodos}

A prevalência da deficiência foi calculada separadamente para homens e mulheres, segundo cor/raça, e segundo o tipo de deficiência e gravidade. Como a estrutura etária segundo cor/raça é diferente, a comparação da prevalência sem padronização pela distribuição etária ficaria comprometida uma vez que existe uma forte relação entre idade e deficiência. Grupos com estruturas etárias mais jovens, como a dos indígenas, poderiam ter a prevalência subestimada enquanto grupos com estruturas etárias mais envelhecidas, como a dos amarelos, poderiam ter a prevalência de deficiência superestimada.

Com o objetivo de controlar a influência da estrutura etária no cálculo da prevalência de deficiências, este estudo utilizou-se da padronização direta, tendo como padrão a estrutura etária da população branca. A taxa padronizada por idade pelo método direto $\left(\mathrm{TB}_{\mathrm{p} . \mathrm{d}}\right)$ é dada por (1):

$$
\mathrm{TB}_{\mathrm{p} . \mathrm{d}}=\sum_{\mathrm{x}} \mathrm{m}_{\mathrm{x}, \mathrm{v}} \cdot \mathrm{Q}_{\mathrm{x}, \mathrm{s}} / \sum_{\mathrm{x}} \mathrm{Q}_{\mathrm{x}, \mathrm{s}}
$$


Em que $\mathrm{m}_{\mathrm{x}, \mathrm{v}}$ representa as taxas específicas, por idade $\mathrm{x}$, da variável da população $\mathrm{v}$, e $\mathrm{Q}_{\mathrm{x}, \mathrm{s}}$ corresponde ao número ou proporção de pessoas de idade $\mathrm{x}$, na população considerada padrão $\mathrm{s} 22$.

Em seguida, para calcular as razões de chances de o indivíduo apresentar alguma deficiência (por tipo e gravidade), segundo cor/raça, foram utilizados modelos de regressão logística multinomial 23. Foi estimado um modelo para cada tipo de deficiência (visual, auditiva e motora), segundo grau de severidade, se grave, leve ou não tem, sendo essa última a categoria de referência.

Como variável explicativa principal, foi utilizada a variável cor/raça, sendo a categoria indígena a referência. Como controle, foram utilizadas as seguintes variáveis: sexo (homens como referência), grupos etários (grupo 60 anos e mais como referência), escolaridade do chefe de domicílio (grupo com até o Ensino Fundamental incompleto como referência), situação do domicílio (rural como referência) e região geográfica (Nordeste como referência).

Como no Censo Demográfico de 2010 foi utilizada uma amostra complexa, foi necessário, para estimar os parâmetros neste estudo, aplicar o comando complex sample do IBM SPSS 25, utilizando as variáveis V0300 (variável controle - clusters), V0011 (área de ponderação - estratos) e V0010 (peso amostral), para correção amostral.

\section{Resultados}

A Tabela 1 apresenta a distribuição percentual das variáveis sexo, escolaridade, situação do domicílio e local de residência, além da idade média dos indivíduos, para aqueles com cinco anos ou mais de idade, segundo cor/raça no Brasil em 2010. Os grupos raciais mais escolarizados são os brancos e amarelos, que também são os que apresentam as maiores idades médias. Já os indígenas são os mais jovens, com uma idade média de 29,6 anos.

Excetuando o grupo de indígenas, os demais são majoritariamente urbanos, com um percentual abaixo de $20 \%$ dos indivíduos residindo no meio rural. Em relação à distribuição regional, os indígenas estão mais presentes no Norte e Nordeste do país. Os brancos se concentram mais no Sul e Sudeste, e pretos, pardos e amarelos, no Sudeste e Nordeste.

As Tabelas 2 e 3 apresentam as prevalências de deficiência (segundo tipo e grau de severidade) para homens e mulheres, respectivamente. São apresentados os resultados não padronizados e padronizados. Ao compará-los, torna-se clara a importância em se padronizar pela estrutura etária ao se fazer comparações de grupos com estruturas diferentes. Após utilizar a estrutura etária da população branca para padronizar as estimativas dos demais grupos de cor/raça, observa-se, nas Tabelas 2 e 3, que as prevalências de deficiência, independentemente do tipo e do grau, mudam consideravelmente.

Os resultados da Tabela 2, para os homens acima de cinco anos, mostram que, antes da padronização, o grupo de cor/raça amarela apresentava a maior prevalência de deficiência visual (19,8\%) e auditiva (6,9\%). Após a padronização, os indígenas são o grupo racial com maior prevalência dessas duas deficiências (20,1\% e 7,1\%, respectivamente). Com relação à deficiência motora, 8,7\% dos indígenas do sexo masculino, com cinco anos ou mais de idade, possuíam deficiência motora grave $(2,8 \%)$ ou leve $(5,9 \%)$, sendo esse novamente o grupo racial com maior prevalência de deficiência.

Entre as mulheres indígenas com cinco anos ou mais, a prevalência de deficiência visual total $(24,8 \%)$ só é superior à prevalência entre as mulheres brancas $(21,9 \%)$ (Tabela 3$)$. Sem a padronização, a prevalência de deficiência visual das indígenas era de 19,4\%, bem abaixo do resultado padronizado. É importante notar que, ao desagregar a prevalência de deficiência visual segundo o grau de severidade, a prevalência de deficiência grave entre as mulheres indígenas com cinco anos ou mais é a maior entre os grupos de cor/raça (6\%). Em relação às demais deficiências, a prevalência é maior entre as mulheres indígenas, independentemente do tipo e grau de severidade.

Finalmente, ao comparar as Tabelas 2 e 3, fica claro que a prevalência de deficiências visual e motora é maior entre as mulheres, já a prevalência de deficiência auditiva é praticamente a mesma entre homens e mulheres, independentemente do grupo de cor/raça.

A Tabela 4 apresenta as razões de chance (RC) de possuir deficiência, segundo tipo e grau de severidade, entre indivíduos com cinco anos ou mais de idade no Brasil em 2010. Os resultados mostram que as diferenças segundo cor/raça são expressivas, mesmo após controlando pelas demais variáveis do modelo. Por um lado, quando se analisa a deficiência visual leve, todas as categorias de cor/raça 
Tabela 1

Distribuição percentual das variáveis segundo cor/raça entre indivíduos com cinco anos e mais de idade. Brasil, 2010.

\begin{tabular}{|c|c|c|c|c|c|}
\hline Variáveis & Branca & Preta & Amarela & Parda & Indígena \\
\hline \multicolumn{6}{|l|}{ Sexo } \\
\hline Homem & 47,7 & 51,8 & 45,2 & 49,6 & 49,9 \\
\hline Mulher & 52,3 & 48,2 & 54,8 & 50,4 & 50,1 \\
\hline Idade média & 35,6 & 34,9 & 35,6 & 31,7 & 29,6 \\
\hline \multicolumn{6}{|l|}{ Escolaridade do chefe do domicílio } \\
\hline Sem instrução/Ensino Fundamental incompleto & 45,4 & 60,8 & 45,9 & 62,3 & 73,2 \\
\hline Ensino Fundamental completo/Ensino Médio incompleto & 15,5 & 15,2 & 14,3 & 14,6 & 11,2 \\
\hline Ensino Médio completo/Ensino Superior incompleto & 25,0 & 19,6 & 24,1 & 18,6 & 12,3 \\
\hline Ensino Superior completo & 14,1 & 4,4 & 15,7 & 4,5 & 3,4 \\
\hline \multicolumn{6}{|l|}{ Situação do domicílio } \\
\hline Urbano & 88,2 & 85,8 & 86,9 & 80,7 & 41,8 \\
\hline Rural & 11,8 & 14,2 & 13,1 & 19,3 & 58,2 \\
\hline \multicolumn{6}{|l|}{ Região geográfica } \\
\hline Norte & 3,9 & 7,1 & 8,2 & 12,8 & 35,4 \\
\hline Nordeste & 16,8 & 34,8 & 29,6 & 38,2 & 26,2 \\
\hline Sudeste & 49,1 & 43,9 & 43,4 & 35,2 & 13,4 \\
\hline Sul & 23,9 & 7,6 & 8,9 & 5,6 & 9,5 \\
\hline Centro-oeste & 6,4 & 6,5 & 9,9 & 8,4 & 15,5 \\
\hline $\mathrm{n}$ & 83.950 .960 & 13.706 .532 & 1.984 .165 & 76.552 .667 & 720.246 \\
\hline $\mathrm{N}$ total & & & 176.914 .570 & & \\
\hline
\end{tabular}

Fonte: Censo Demográfico de 201018.

Tabela 2

Prevalência da deficiência, entre homens, segundo cor/raça, entre indivíduos com cinco anos e mais de idade. Brasil, 2010.

\begin{tabular}{|c|c|c|c|c|c|c|c|c|c|}
\hline \multirow[t]{3}{*}{ Deficiência } & \multirow[t]{3}{*}{ Branca } & \multicolumn{2}{|c|}{ Preta } & \multicolumn{2}{|c|}{ Amarela } & \multicolumn{2}{|c|}{ Parda } & \multicolumn{2}{|c|}{ Indígena } \\
\hline & & Não & Padronizado & Não & Padronizado & Não & Padronizado & Não & Padronizado \\
\hline & & padronizado & & padronizado & & padronizado & & padronizado & \\
\hline \multicolumn{10}{|l|}{ Visual } \\
\hline Grave & 2,9 & 3,7 & 4,0 & 3,5 & 3,4 & 3,1 & 3,7 & 3,5 & 4,6 \\
\hline Leve & 14,0 & 14,9 & 15,6 & 16,3 & 16,1 & 14,0 & 16,1 & 12,3 & 15,6 \\
\hline Total & 16,9 & 18,6 & 19,5 & 19,8 & 19,5 & 17,1 & 19,8 & 15,7 & 20,1 \\
\hline \multicolumn{10}{|l|}{ Auditiva } \\
\hline Grave & 1,4 & 1,3 & 1,4 & 1,6 & 1,5 & 1,2 & 1,5 & 1,2 & 1,5 \\
\hline Leve & 4,5 & 4,5 & 4,8 & 5,3 & 5,0 & 4,2 & 5,0 & 4,4 & 5,6 \\
\hline Total & 5,8 & 5,7 & 6,2 & 6,9 & 6,4 & 5,4 & 6,5 & 5,6 & 7,1 \\
\hline \multicolumn{10}{|l|}{ Motora } \\
\hline Grave & 1,9 & 2,3 & 2,6 & 2,0 & 1,9 & 1,9 & 2,4 & 2,1 & 2,8 \\
\hline Leve & 3,6 & 4,5 & 4,8 & 4,1 & 3,9 & 3,7 & 4,6 & 4,5 & 5,9 \\
\hline Total & 5,5 & 6,8 & 7,4 & 6,1 & 5,8 & 5,6 & 7,0 & 6,6 & 8,7 \\
\hline
\end{tabular}

Fonte: Censo Demográfico de 201018. 
Tabela 3

Prevalência da deficiência, entre mulheres, segundo cor/raça, entre indivíduos com cinco anos e mais de idade. Brasil, 2010.

\begin{tabular}{|c|c|c|c|c|c|c|c|c|c|}
\hline \multirow[t]{3}{*}{ Deficiência } & \multirow[t]{3}{*}{ Branca } & \multicolumn{2}{|c|}{ Preta } & \multicolumn{2}{|c|}{ Amarela } & \multicolumn{2}{|c|}{ Parda } & \multicolumn{2}{|c|}{ Indígena } \\
\hline & & Não & Padronizado & Não & Padronizado & Não & Padronizado & Não & Padronizado \\
\hline & & padronizado & & padronizado & & padronizado & & padronizado & \\
\hline \multicolumn{10}{|l|}{ Visual } \\
\hline Grave & 4,0 & 5,5 & 5,8 & 4,6 & 4,8 & 4,4 & 5,4 & 4,0 & 6,0 \\
\hline Leve & 18,0 & 20,7 & 21,2 & 20,7 & 21,1 & 19,1 & 21,7 & 14,9 & 18,8 \\
\hline Total & 21,9 & 26,2 & 27,1 & 25,3 & 25,9 & 23,5 & 27,1 & 19,4 & 24,8 \\
\hline \multicolumn{10}{|l|}{ Auditiva } \\
\hline Grave & 1,2 & 1,2 & 1,3 & 1,2 & 1,3 & 1,0 & 1,3 & 1,1 & 1,5 \\
\hline Leve & 4,1 & 4,7 & 5,0 & 4,8 & 5,0 & 4,1 & 5,0 & 4,4 & 5,7 \\
\hline Total & 5,3 & 5,9 & 6,3 & 6,1 & 6,3 & 5,1 & 6,4 & 5,6 & 7,2 \\
\hline \multicolumn{10}{|l|}{ Motora } \\
\hline Grave & 3,0 & 3,8 & 4,2 & 2,8 & 2,9 & 2,8 & 3,8 & 3,0 & 4,0 \\
\hline Leve & 5,9 & 7,7 & 8,2 & 6,3 & 6,6 & 6,1 & 7,8 & 6,5 & 8,8 \\
\hline Total & 8,9 & 11,5 & 12,4 & 9,1 & 9,5 & 8,8 & 11,5 & 9,5 & 13,0 \\
\hline
\end{tabular}

Fonte: Censo Demográfico de 201018

apresentam maior chance de possuir tal deficiência do que os indígenas. Destaque deve ser dado aos pardos, que possuem $78 \%$ (valor de $\mathrm{p}<0,001$ ) a mais de chance de ser deficiente visual leve do que os indígenas. Já os brancos apresentam menor chance de ter deficiência visual grave $(\mathrm{RC}=0,84$; valor de $\mathrm{p}<0,001)$ que os indígenas, enquanto os pretos têm maior chance $(\mathrm{RC}=1,09$; valor de $\mathrm{p}<0,001)$ que o grupo de referência.

Por outro lado, indígenas apresentam maior chance de possuir deficiência auditiva, leve ou grave, quando comparados aos demais grupos de cor/raça. A única exceção é observada ao compará-los aos amarelos. Já, quando se analisa os resultados para deficiência motora, a desvantagem da população indígena fica muito evidente. Todos os grupos de cor/raça, exceto os pretos, apresentam uma chance bem inferior aos dos indígenas de possuir deficiência motora, leve ou grave. Os brancos, por exemplo, apresentam $25 \%$ (valor de $\mathrm{p}<0,001$ ) a menos de chance que os indígenas de possuir deficiência motora leve e $22 \%$ (valor de p $<0,001$ ) a menos de chance, quando se analisa a deficiência motora grave.

Os resultados segundo sexo mostram que as mulheres, após controlar pelas demais variáveis do modelo, apresentam maior chance de ter deficiência visual e motora, grave e leve, em relação aos homens. Já, ao analisar a dificuldade grave e leve de audição, a desvantagem passa a ser masculina, ou seja, a chance é menor entre as mulheres.

Os resultados para as demais variáveis dos modelos confirmam que idade e escolaridade do responsável pelo domicílio têm uma associação muito forte com a chance de possuir uma deficiência (grave ou leve). Como esperado, quanto mais velho o grupo etário, maior é a chance de apresentar uma deficiência, especialmente ao se analisar a deficiência motora. E, quanto maior a escolaridade do responsável pelo domicílio, menor a chance de apresentar um dos três tipos de deficiência examinados neste trabalho, independentemente de sua gravidade. Indivíduos que residem em domicílios cujo responsável tem Ensino Superior completo, por exemplo, apresentam uma chance muito inferior de possuir alguma deficiência, grave ou leve, do que indivíduos que vivem em domicílios cujo responsável não tem instrução ou tem o Ensino Fundamental incompleto.

Finalmente, indivíduos que moram na área urbana têm uma maior chance de reportar qualquer uma das três deficiências, independentemente do grau. Residentes do Norte, Sudeste, Sul e Centro-oeste apresentam uma chance maior que aqueles residentes no Nordeste do país, especialmente quando se analisa as deficiências visual e auditiva. 
Tabela 4

Razão de chance de apresentar deficiência, segundo tipo e gravidade. Brasil, 2010.

\begin{tabular}{|c|c|c|c|c|c|c|}
\hline \multirow[t]{3}{*}{ Variáveis } & \multicolumn{2}{|c|}{ Deficiência visual } & \multicolumn{2}{|c|}{ Deficiência auditiva } & \multicolumn{2}{|c|}{ Deficiência motora } \\
\hline & Grave & Leve & Grave & Leve & Grave & Leve \\
\hline & \multicolumn{2}{|c|}{$\operatorname{Exp}(B)$} & \multicolumn{2}{|c|}{$\operatorname{Exp}(B)$} & \multicolumn{2}{|c|}{$\operatorname{Exp}(B)$} \\
\hline (Intercept) & $0,23 *$ & 0,53 * & 0,09 * & 0,27 * & 0,18 * & 0,33 * \\
\hline \multicolumn{7}{|l|}{ Cor/Raça } \\
\hline Indígena (Ref.) & 1,00 & 1,00 & 1,00 & 1,00 & 1,00 & 1,00 \\
\hline Branca & 0,84 * & 1,07 * & 0,99 & 0,88 * & 0,78 * & 0,75 * \\
\hline Preta & 1,09 * & 1,20 * & $0,91 * *$ & 0,92 * & 0,97 & $0,97 * * *$ \\
\hline Amarela & 1,03 & 1,28 * & $1,08 * *$ & 1,03 & 0,75 * & 0,82 * \\
\hline Parda & 0,97 & 1,78 * & $0,92 * *$ & 0,93 * & 0,83 * & 0,86 * \\
\hline \multicolumn{7}{|l|}{ Sexo } \\
\hline Homem (Ref.) & 1,00 & 1,00 & 1,00 & 1,00 & 1,00 & 1,00 \\
\hline Mulher & 1,48 * & $1,41 *$ & $0,79 *$ & 0,88 * & 1,55 * & 1,68 * \\
\hline \multicolumn{7}{|l|}{ Grupo etário (anos) } \\
\hline 60 e mais (Ref.) & 1,00 & 1,00 & 1,00 & 1,00 & 1,00 & 1,00 \\
\hline $5-14$ & 0,05 * & 0,10 * & 0,06 * & 0,06 * & 0,02 * & 0,02 * \\
\hline $15-59$ & 0,17 * & 0,30 * & 0,13 * & 1,16 * & $0,09 *$ & 0,13 * \\
\hline \multicolumn{7}{|l|}{ Local de residência } \\
\hline Rural (Ref.) & 1,00 & 1,00 & 1,00 & 1,00 & 1,00 & 1,00 \\
\hline Urbano & 1,08 * & $1,07 *$ & 1,06 * & $1,04 *$ & 1,26 * & 1,06 * \\
\hline \multicolumn{7}{|l|}{ Região } \\
\hline Nordeste (Ref.) & 1,00 & 1,00 & 1,00 & 1,00 & 1,00 & 1,00 \\
\hline Norte & 1,47 * & $1,41 *$ & $1,21 *$ & 1,24 * & $1,09 *$ & $1,20 *$ \\
\hline Sudeste & $1,61 *$ & 1,34 * & $1,01 *$ & $1,11 *$ & 1,13 & 1,17 \\
\hline Sul & 1,23 * & $1,29 *$ & $0,94 \#$ & 1,18 * & 0,98 & 1,02 \\
\hline Centro-oeste & 1,35 * & 1,09 * & 1,18 * & 1,20 * & 1,15 * & 1,02 \\
\hline \multicolumn{7}{|l|}{ Escolaridade do chefe de domicílio } \\
\hline Ensino Fundamental incompleto (Ref.) & 1,00 & 1,00 & 1,00 & 1,00 & 1,00 & 1,00 \\
\hline Ensino Médio incompleto & 0,64 * & 0,80 * & 0,67 * & 0,76 * & $0,60 *$ & $0,67 *$ \\
\hline Ensino Superior incompleto & 0,50 * & 0,70 * & $0,54 *$ & 0,64 * & 0,46 * & $0,51 *$ \\
\hline Ensino Superior completo & 0,38 * & 0,65 * & 0,42 * & 0,53 * & 0,33 * & 0,38 * \\
\hline
\end{tabular}

Ref.: referência.

Fonte: Censo Demográfico de 201018.

* $\mathrm{p}=0,001$;

** $p=0,05$.

$\star \star * \mathrm{p}=0,1$

$\# p=0,01$.

\section{Discussão}

O objetivo deste trabalho foi estimar a prevalência de deficiências visual, auditiva e motora para a população indígena, segundo grau de severidade, e compará-la (utilizando a padronização direta) com aquelas observadas nos demais grupos de cor/raça do Brasil em 2010. Em seguida, este trabalho estimou a chance de ter uma das três deficiências, separadamente e por grau de severidade, para cada grupo de cor/raça em relação aos indígenas, ao mesmo tempo em que outras variáveis associadas à chance de apresentar deficiência foram controladas.

Os resultados padronizados mostram que os indígenas, tanto homens quanto mulheres, apresentam a maior prevalência em cada uma das deficiências examinadas neste trabalho, sendo a única 
exceção a deficiência visual de grau leve entre as mulheres. Resultados não padronizados escondem tal desvantagem da população indígena no Brasil e sugerem, erroneamente, que a prevalência de deficiência visual, auditiva e motora dos indígenas é, em alguns casos, menor que a da população branca. Essa comparação equivocada ocorre quando grupos com diferentes estruturas etárias, como os grupos de cor/raça no Brasil, são comparados sem a utilização de uma estrutura etária padrão. Dessa maneira, os resultados padronizados apresentados neste trabalho contribuem para uma comparação adequada da prevalência de deficiências visual, auditiva e motora entre grupos de cor/raça no Brasil.

A maior prevalência de deficiência física entre a população indígena tem sido observada no cenário internacional. Estudos australianos apresentam dados semelhantes aos encontrados por este trabalho. Segundo DiGiacomo et al. 24, a chance de os indígenas australianos apresentarem alguma deficiência é uma vez e meia maior que a dos não indígenas. Similarmente, Durst \& Bluechardt 25 revelam que, no Canadá, a prevalência da deficiência auditiva e visual é bastante superior a observada entre os não indígenas. Já a deficiência motora é praticamente a mesma entre os dois grupos. Na Nova Zelândia, segundo Hickey \& Wilson 26, também é identificado uma maior prevalência de deficiência entre os indígenas, quando comparados com os não indígenas. Nos Estados Unidos, em 2012, quase $18 \%$ da população indígena adulta apresentava algum tipo de deficiência, um valor $72 \%$ maior que a prevalência entre os brancos 17 .

Neste trabalho, ao analisar os resultados dos modelos de regressão multinomial, a desvantagem da população indígena em relação a um ou mais grupos de cor/raça se mantém segundo todas as deficiências analisadas, exceto entre aqueles que reportaram leve dificuldade de enxergar. Esse resultado pode ser parcialmente explicado por potenciais diferenças na percepção da deficiência entre grupos de cor/raça.

No Censo Demográfico brasileiro, a presença ou ausência de deficiência é uma informação de saúde coletada pelo entrevistador junto ao morador residente do domić́lio que esteja no momento da visita pelo entrevistado que se encontra no domicílio, que responde por ele e pelos demais residentes do domicílio. Estudos na área da saúde recorrentemente alertam que, pelo menos, dois aspectos devem ser considerados ao analisar dados em pesquisas domiciliares: (1) a subjetividade inerente à autoavaliação (dependente de fatores demográficos, sociais, econômicos e contextuais) 27,28 e (2) o papel do respondente substituto 29 .

O primeiro aspecto depende da percepção do indivíduo sobre sua saúde, que pode variar segundo diferentes fatores, como, por exemplo, o sexo, a idade e a cor/raça do indivíduo. Ao comparar os grupos de cor/raça diferentes, os resultados deste trabalho podem estar subestimados ou superestimados. O segundo fator deriva do fato de que o respondente pode não ser o indivíduo que possui a deficiência, mas sim outro residente do domicílio que respondeu ao questionário do Censo. Nesse caso, a avaliação passa a ser feita por outra pessoa, e não pelo portador da deficiência. A percepção do respondente substituto também pode comprometer os resultados e as comparações segundo cor/raça.

Apesar dessas ressalvas, os resultados encontrados neste estudo vão ao encontro dos resultados internacionais já citados anteriormente. De uma maneira geral, a situação dos indígenas é pior do que a de outros grupos de cor/raça, mesmo em países altamente desenvolvidos.

Este trabalho contribui para um tema ainda pouco analisado pela literatura brasileira sobre a saúde dos povos indígenas. Na verdade, diferentes estudos que analisam fatores associados à prevalência de deficiência física na população brasileira não consideram a variável cor/raça ou, quando o fazem, excluem a categoria da população indígena da análise 19,20.

\section{Conclusão}

Os resultados deste estudo evidenciam mais uma desvantagem na saúde dos povos indígenas no Brasil. A prevalência de deficiência visual, auditiva e motora é quase sempre a maior nesse grupo populacional. Um aspecto importante a se destacar é a impossibilidade de acessar as informações sobre Terras Indígenas, língua falada e etnias, presentes no Censo Demográfico de 2010. Tal acesso possibilitaria uma análise mais pormenorizada, considerando, por exemplo, a etnia. Tal impedimento empobrece as análises e impossibilita possíveis associações entre aspectos culturais e deficiências, tão reclamados pela literatura internacional 16,24,25. 
Apesar dessas limitações, esses primeiros resultados, por si, já apontam para diversas possibilidades de pesquisa, seja utilizando o próprio Censo Demográfico, seja incentivando pesquisas de campo para compreender melhor os sentidos e significados das deficiências entre os povos indígenas. Esse grupo populacional experimenta questões e desafios que os brancos, por exemplo, nem sempre enfrentam, como aspectos relacionados à opressão cultural e racismo 26.

\section{Colaboradores}

C. S. Dias Junior contribuiu na concepção geral, análise dos dados, redação e revisão final do trabalho. A. P. Verona participou nas análises dos dados, na redação e revisão final do trabalho.

\section{Referências}

1. Simões BS, Machado-Coelho GLL, Pena JL, Freitas SN. Condições ambientais e prevalência de infecção parasitária em indígenas $\mathrm{Xu}$ kuru-Kariri, Caldas, Brasil. Rev Panam Salud Pública 2015; 38:42-8.

2. Dias Júnior CS, Oliveira CR, Verona AP, Pena JL, Sírio MA, Bahia MT, et al. Prevalência de parasitoses intestinais e estado nutricional, segundo sexo e idade, entre a população indígena Caxixó, Minas Gerais, Brasil. Rev Bras Estud Popul 2013; 30:603-8.

3. Freitas GA, Souza MCC, Lima RC. Prevalência de diabetes mellitus e fatores associados em mulheres indígenas do Município de Dourados, Mato Grosso do Sul, Brasil. Cad Saúde Pública 2016; 32:e00023915.

4. Bresan D, Bastos JL, Leite MS. Epidemiology of high blood pressure among the Kaingang people on the Xapecó Indigenous Land in Santa Catarina State, Brazil. Cad Saúde Pública 2013; 31:331-44.

5. Boaretto JD, Molena-Fernandes C, Pimentel GGA. Estado nutricional de indígenas Kaingang e Guarani no Estado do Paraná, Brasil. Ciênc Saúde Coletiva 2015; 20:2323-8.

6. Fávaro TR, Santos RV, Cunha GM, Leite IC, Coimbra Jr. CEA. Obesidade e excesso de peso em adultos indígenas Xukuru do Ororubá, Pernambuco, Brasil: magnitude, fatores socioeconômicos e demográficos associados. Cad Saúde Pública 2015; 31:1685-97. 
7. Mauricio HA, Moreira RS. Oral health status of the ethnic group Xukuru from Ororubá: multilevel analysis. Rev Bras Epidemiol 2014; 17:787-800

8. Alves Filho P, Santos RV, Vettore MV. Saúde bucal dos índios Guarani no Estado do Rio de Janeiro, Brasil. Cad Saúde Pública 2009; 25:3746.

9. Souza MLP, Garnelo L, Deslandes SF. Modos de vida e modos de beber de jovens indígenas em um contexto de transformações. Ciênc Saúde Coletiva 2010; 15:709-16.

10. Souza MLP, Garnelo L. Quando, como e o que se bebe: o processo de alcoolização entre populações indígenas do alto Rio Negro, Brasil. Cad Saúde Pública 2007; 23:1640-8.

11. Oliveira CS, Lotufo Neto F. Suicídio entre povos indígenas: um panorama estatístico brasileiro. Rev Psiquiatr Clín (São Paulo) 2003; 30:4-10.

12. Campos MB, Borges GM, Queiroz BL, Santos RV. Diferenciais de mortalidade entre indígenas e não indígenas no Brasil com base no Censo Demográfico de 2010. Cad Saúde Pública 2017; 33:e00015017.

13. Santos RV, Coimbra Jr. CEA, Escobar AL. Epidemiologia e saúde dos povos indígenas no Brasil. Rio de Janeiro: Editora Fiocruz; 2003.

14. King M, Smith A, Gracey M. Indigenous health part 2: the underlying causes of the health gap. Lancet 2009; 374:76-85.

15. Ng E. Disability among Canada's aboriginal peoples in 1991. Health Rep 1996; 8:25-32.

16. Gilroy J, Colmar S, Donelly M, Parmenter T. Conceptual framework for policy and research development with Indigenous persons with disability. Australian Aboriginal Studies 2013; 2:42-58.

17. Erickson W, Lee C, von Schrader S. 2012 disability status report: United States. Ithaca: Yang-Tan Institute on Employment and Disability, Cornell University; 2014.

18. Instituto Brasileiro de Geografia e Estatística. Censo Demográfico de 2010: características gerais da população, religião e deficiência. Rio de Janeiro: Instituto Brasileiro de Geografia e Estatística; 2012.

19. Malta DC, Stopa SR, Canuto R, Gomes NL, Mendes VLF, Goulart BNG, et al. Prevalência autorreferida de deficiência no Brasil, segundo a Pesquisa Nacional de Saúde, 2013. Ciênc Saúde Coletiva 2016, 21:3253-64.
20. Castro SS, César CLG, Carandina L, Barros MBA, Alves MCGP, Goldbaum M. Deficiência visual, auditiva e física: prevalência e fatores associados em estudo de base populacional. Cad Saúde Pública 2008; 24:1773-82.

21. Secretaria Nacional de Promoção dos Direitos da Pessoa com Deficiência. Cartilha do censo 2010: pessoas com deficiência. Brasília: Secretaria Nacional de Promoção dos Direitos da Pessoa com Deficiência, Secretaria de Direitos Humanos da Presidência da República; 2012.

22. Carvalho JAM, Sawyer DO, Rodrigues RN. Introdução a alguns conceitos básicos e medidas em demografia. São Paulo: Associação Brasileira das Empresas de Pesquisas; 1998.

23. Homer Jr. DW, Lemeshow S, Sturdivant RX. Applied logistic regression. 3rd Ed. New York: John Wiley and Sons; 2013. (Wiley Series in Probability and Statistics).

24. DiGiacomo M, Davidson PM, Abbott P, Delaney $\mathrm{P}$, Dharmendra T, McGrath SJ, et al. Childhood disability in aboriginal and Torres Strait Islander peoples: a literature review. Int J Equity Health 2013; 12:1-18.

25. Durst D, Bluechardt M. Aboriginal people with disabilities: a vacuum in public policy. Saskatchewan: the Saskatchewan Institute of Public Policy; 2004 (SIPP Briefing Note, Issue 6).

26. Hickey H, Wilson D. Whanau Haua: reframing disability from an indigenous perspective. MAI Journal 2017; 6:83-94.

27. Szwarcwald CL, Souza Junior PRB, Esteves MAP, Damacena GN, Viacava F. Socio-demographic determinants of self-rated health in Brazil. Cad Saúde Pública 2005; 21 Suppl 1:S54-64.

28. Cruz MS, Lima MCP, Santos JLF, Duarte YAO, Lebrão ML, Ramos-Cerqueira ATAR. Deficiência auditiva referida por idosos no Município de São Paulo, Brasil: prevalência e fatores associados (Estudo SABE, 2006). Cad Saúde Pública 2012; 28:1479-92.

29. Lima-Costa MF, Peixoto SV, Firmo JOA, Uchôa E. A influência de respondente substituto na percepção da saúde de idosos: um estudo baseado na Pesquisa Nacional por Amostra de Domicílios (1998, 2003) e na coorte de Bambuí, Minas Gerais, Brasil. Cad Saúde Pública 2007; 23:1893-902. 


\section{Abstract}

This study aimed to describe the prevalence of visual, hearing, and motor impairments in the indigenous population in Brazil and to estimate the odds of presenting one of the three disabilities, separately, according to degree of severity. The data were obtained from the 2010 Population Census conducted by the Brazilian Institute of Geography and Statistics (IBGE). The methods included direct standardization for calculation of the prevalence rates and multinomial logistic regression models. According to the standardized results, indigenous men and women showed the highest prevalence in each of the three disabilities, except for mild visual impairment in women. The results of the multinomial regression models revealed a relative disadvantage for indigenous peoples in nearly all the types of disability.

Disable Persons; South American Indians; Health of Indigenous Peoples

\section{Resumen}

El objetivo de este estudio fue describir la prevalencia de discapacidad visual, auditiva y motora y estimar la oportunidad de sufrir una de las tres discapacidades, separadamente, según el grado de severidad, en la población indígena de Brasil. Los datos se obtuvieron del Censo Demográfico de 2010, recogidos por el Instituto Brasileño de Geografía y Estadística (IBGE). Los métodos utilizados incluyen la estandarización directa para el cálculo de las prevalencias y modelos de regresión logística multinomial. Los resultados estandarizados muestran que hombres y mujeres indígenas presentan la mayor prevalencia en cada una de las discapacidades examinadas en este trabajo, siendo la única excepción la discapacidad visual de grado leve entre las mujeres. Los resultados de los modelos de regresión multinomial muestran una desventaja relativa de los pueblos indígenas en casi todos los tipos de discapacidad.

Personas con Discapacidad; Indios

Sudamericanos; Salud de Poblaciones

Indigenas
Recebido em 01/Ago/2017

Versão final reapresentada em 30/Abr/2018

Aprovado em 14/Mai/2018 